To understand the mechanisms at play, the researchers used density functional theory in conjunction with transmission electron microscopy to study the structure and electrodynamics of the domain walls. Calculating the layer-by-layer polarization, they found a discontinuity in the electrostatic potential across conducting walls and theorized that extra carriers may accumulate to compensate this step. Analysis of the electronic struc- ture also revealed changes in the bandgap near conducting domain walls. Both effects are absent at non-conducting walls, therefore the researchers proposed that the combination of band structure changes and increased carrier concentration enable the observed conductivity.

The researchers also demonstrated a proof-of-concept for using these conduction channels in a device. They grew mono-domain BFO between in-plane electrodes (see Figure 1) and drew domain walls between them using PFM, showing that the conductivity across the BFO scales linearly with the number of channels drawn. They showed that the process is reversible by erasing a channel and recovering the previous conductivity, suggesting the possibility of a dynamic logic or memory element that could be reconfigured with tremendous precision.

AlisOn HaTt

\section{Self-Assembled Chiral Capsules Exhibit Highly Polar Interiors}

The catalytic function of enzymes relies on the protein structure sequestering polar active sites from the bulk solution, creating a specific environment. Capsule molecules synthesized to mimic this environment can also serve as molecular containers or nanoreactors but their interiors are typically achiral and nonpolar, which limits their selectivity and activity. In previously reported cases where the capsule interiors were both polar and chiral, encapsulation of guest molecules was mainly hydrophobic because polar groups were too exposed to interactions with solvent. Recently, however, B. Kuberski and A. Szumna at the Institute of Organic Chemistry, Polish Academy of Sciences, Warsaw, synthesized with a one- or twostep process self-complimentary homochiral and heterochiral resorcinarene capsules with buried polar groups that can encapsulate highly polar guests.

As reported earlier this year in Chemical Communications (DOI: 10.1039/b820990a), Kuberski and Szumna performed a Mannich reaction with resorcin[4]arene and L-phenylalanine under mild conditions that allowed the isolation of secondary amine L-1 (see Figure 1) in high yield. The researchers said that the high solubility displayed by L-1 in $\mathrm{CDCl}_{3}$ $(>70 \mathrm{mg} / \mathrm{ml})$ and its sharp concentrationindependent ${ }^{1} \mathrm{H}$ and ${ }^{13} \mathrm{C}$ nuclear magnetic resonance (NMR) spectra can only be explained by burial of the polar functionalities in the interior of the molecule, together with a highly symmetrical con- formation. The complementary amine and acid functionalities led the researchers to consider a dimeric capsular structure, $(\mathrm{L}-1)_{2}$, with $D_{4}$ symmetry, which they confirmed with $x$-ray analysis. Sealing the capsular structure are two seams of salt bridges formed between amine and carboxyl groups, as well as numerous hydrogen bonds. All interior polar func-

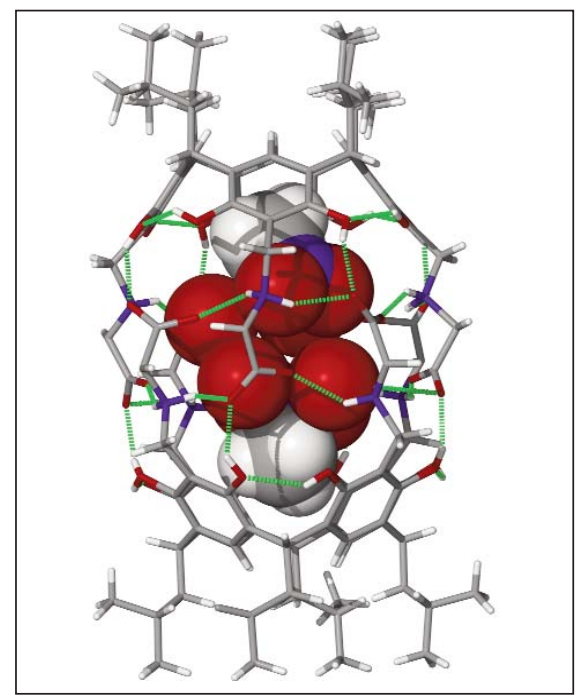

Figure 1. The $x$-ray structure of $(\mathrm{L}-\mathbf{1})_{2}$ shows the capsule skeleton (side chains are omitted for clarity) with encapsulated $\left(\mathrm{MeNO}_{2}\right)_{2}\left(\mathrm{H}_{2} \mathrm{O}\right)_{4}$. The red spheres are oxygen; dark gray, carbon; and light gray, hydrogen. Reprinted by permission from Chemical Communications (2009) (DOI: 10.1039/b820990a); C2009 Royal Chemical Society. tionalities are isolated from the bulk solvent by hydrophobic phenylalanine side chains. Filling the capsule, whose volume was calculated to be $310 \AA^{3}$, are two nitromethane molecules, which $\mathrm{H}$-bond to the host amino groups, and four water molecules, which the researchers suggest $\mathrm{H}$-bond to host acid moieties.

The researchers expected increased stability from a heterocyclic capsule with $S_{8}$ symmetry (symmetry associated with rotation by $360^{\circ} / 8=45^{\circ}$ followed by reflection through a plane perpendicular to the $S_{8}$ axis) so they mixed (L-1) $)_{2}$ with its mirror image isomer, (D-1) 2 . NMR spectroscopy showed quantitative formation of the heterochiral dimer, (D-1)( L-1), but only after several dissolve-evaporate cycles or treatment with $10 \% \mathrm{MeOH}(>8 \mathrm{~h}$ ) followed by redissolution in $\mathrm{CDCl}_{3}$, showing that the heterochiral dimer is thermodynamically much more stable than the homochiral dimer. The researchers further demonstrated the existence of dimeric species in solution with diffusion NMR spectroscopy. While the researchers found indirect evidence for water encapsulation in each dimer, they detected ethanol encapsulation with NMR. Kuberski and Szumna said, "Our preliminary complexation studies indicate that $(\mathrm{L}-\mathbf{1})_{2}$ is able to extract from the aqueous phase and encapsulate biologically relevant polar molecules (e.g., one or two molecules of hydroxyacids) and differentiate between enantiomers. Detailed studies on recognition and possible catalytic functions are currently under way."

STEVEN TROHALAKI

\section{Modeling of Hierarchical Nanostructures in Proteins Reveals Structure-Property Relationship}

Modeling the multiscale behavior of protein assemblies with the goal of elucidating the relationship between structure and materials properties represents a challenge at the interface of materials science and biology. Markus Buehler of the Massachusetts Institute of Technology (MIT) and Theodor Ackbarow of MIT and the Max Planck Institute of Colloids and Interfaces, who study the structure of protein-based materials with the aim of learning the key to their lightweight and robust strength, have discovered that the particu- lar arrangement of proteins that produces the sturdiest product is not the arrangement with the most built-in redundancy or the most complicated pattern. Instead, the optimal arrangement of proteins in the ropelike structures they studied is a repeated pattern of two stacks of four bundled alpha-helical proteins. 